\title{
BMJ Open Management of chronic headache with referral from primary care to direct access to MRI compared with Neurology services: an observational prospective study in London
}

\author{
Tiago Rua (D) , ${ }^{1,2}$ Asif Mazumder, ${ }^{3}$ Yvonne Akande, $^{2}$ Charikleia Margariti, ${ }^{2}$ \\ Juliana Ochulor, ${ }^{4}$ Joanna Turville, ${ }^{2}$ Reza Razavi, ${ }^{5}$ Janet L Peacock, ${ }^{6}$ Paul McCrone, \\ Vicky Goh, ${ }^{7}$ James Shearer, ${ }^{6}$ Shazia Afridi ${ }^{4}$
}

To cite: Rua T, Mazumder A, Akande $\mathrm{Y}$, et al. Management of chronic headache with referral from primary care to direct access to MRI compared with Neurology services: an observational prospective study in London. BMJ Open 2020;10:e036097. doi:10.1136/ bmjopen-2019-036097

- Prepublication history and supplemental material for this paper are available online. To view these files, please visit the journal online (http://dx.doi. org/10.1136/bmjopen-2019036097).

Received 02 December 2019 Revised 22 June 2020 Accepted 25 June 2020

A Check for updates

(C) Author(s) (or their employer(s)) 2020. Re-use permitted under CC BY-NC. No commercial re-use. See rights and permissions. Published by BMJ.

For numbered affiliations see end of article.

Correspondence to

Tiago Rua; tiago.rua@kcl.ac.uk

\section{ABSTRACT}

Objectives To evaluate the cost, accessibility and patient satisfaction implications of two clinical pathways used in the management of chronic headache.

Intervention Management of chronic headache following referral from Primary Care that differed in the first appointment, either a Neurology appointment or an MRI brain scan.

Design and setting A pragmatic, non-randomised,

prospective, single-centre study at a Central Hospital in London.

Participants Adult patients with chronic headache referred from primary to secondary care.

Primary and secondary outcome

measures Participants' use of healthcare services and costs were estimated using primary and secondary care databases and questionnaires quarterly up to 12 months postrecruitment. Cost analyses were compared using generalised linear models. Secondary outcomes assessed: access to care, patient satisfaction, headache burden and self-perceived quality of life using headache-specific (Migraine Disability Assessment Scale and Headache Impact Test) and a generic questionnaire (5-level EQ-5D). Results Mean (SD) cost up to 6 months postrecruitment per participant was $£ 578$ (£420) for the Neurology group $(n=128)$ and $£ 245$ ( $£ 172)$ for the MRI group $(n=95)$, leading to an estimated mean cost difference of $£ 333(95 \% \mathrm{Cl} £ 253$ to $£ 413, \mathrm{p}<0.001)$. The mean cost difference at 12 months increased to $£ 518(95 \% \mathrm{Cl} £ 401$ to $£ 637, p<0.001)$. When adjusted for baseline and followup imbalances between groups, this remained statistically significant. The utilisation of brain MRI improved access to care compared with the Neurology group $(p<0.001)$. Participants in the Neurology group reported higher levels of satisfaction associated with the pathway and led to greater change in care management.

Conclusion Direct referral to brain MRI from Primary Care led to cost-savings and quicker access to care but lower satisfaction levels when compared with referral to Neurology services. Further research into the use of brain MRI for a subset of patient population more likely to be reassured by a negative brain scan should be considered. Trial registration number NCT02753933.
Strengths and limitations of this study

- The estimate of healthcare resource use was based on comprehensive and complete data retrieved from hospital databases supplemented by both primary care utilisation data and self-reported participant data.

- The evaluation of the intervention's impact considered different dimensions of analysis (efficiency, quality of care, access to care and patient satisfaction).

- This was a single-centre study with participants recruited from one central hospital in London, therefore a multicentre study would be necessary to explore the generalisability of the results.

\section{INTRODUCTION}

Globally, the percentage of the adult population with an active headache disorder is $47 \%$ for episodic headache and $3 \%$ for chronic headache (lasting more than 15 days per month). ${ }^{1}$ Headache is in the top 10 international causes of disability, ${ }^{2}$ with nearly half of sufferers reporting it affects work, home or social activities. ${ }^{3-5}$ Most headaches are primary headache disorders, such as migraine or tension-type headaches. Secondary headaches, due to an underlying serious pathology (eg, tumour, brain aneurysm) are far less common. ${ }^{6}$ In fact, less than $0.1 \%$ of the lifetime headache prevalence is associated with a life-threatening condition, which can include primary or secondary brain tumours. ${ }^{23}$

Most headache sufferers self-manage, but over $4 \%$ of adults each year consult their general practitioner (GP). ${ }^{78}$ GPs manage $97 \%$ of headache presentations, particularly GPs with special interest and training in headache training initiatives, ${ }^{9}$ with $2 \%$ of 
these referred to neurologists and $1 \%$ to other specialists. ${ }^{7}$ Headache is the most common cause for GP referrals to neurologists accounting for up to $20 \%-30 \%,{ }^{10-13}$ the vast majority of these are for migraine. Chronic migraine sufferers ( $>15$ days/month) had more emergency department (ED)/hospital visits, and diagnostic tests than those with episodic migraine and consequently, the medical costs were three times higher. ${ }^{14}$ Hence, despite the benign nature of most headaches, headache management is associated with high healthcare utilisation. Furthermore, a study in multiple countries of the European Union found that headache management was variable in terms of visits with GPs and specialists and medication use. ${ }^{15}$ Between 2012 and 2014, patients presenting to headache specialists (either neurologists or GPs with a special interest in headache) costed $£ 956$ million in the UK. ${ }^{13} 16$

GP direct access to imaging has been defined as a priority within the National Health Service (NHS), with direct access to brain MRI for the diagnosis of brain cancer identified as a specific initiative. ${ }^{8}$ GPs have reported referrals for secondary care, both for a neurologist consultation or neuroimaging, when they were unable to reassure the patient. ${ }^{17} 18$ Furthermore, Morgan et al found that referral for headache is often the outcome of patient pressure and anxiety interacting with GP characteristics, organisational factors and service availability rather than the headache severity itself. ${ }^{17}$ This contrasts with the recommendations from the National Institute for Health and Care Excellence that does not recommend the use of neuroimaging for reassurance purposes. ${ }^{19}$ A US study estimated that patients with new onset migraine headache or a flare-up of chronic headache had, respectively, a $39 \%$ (95\% CI $24 \%$ to $54 \%$ ) and $51 \%$ (95\% CI $32 \%$ to $68 \%$ ) probability of having neuroimaging routinely ordered even where guidelines specifically recommended against this approach. ${ }^{20} \mathrm{~A}$ UK-based randomised controlled trial evaluated the cost implications of using brain MRI for reassurance purposes and found that it led to a decrease in healthcare costs, in particular for patients with higher levels of psychiatric morbidity. ${ }^{21}$

In summary, despite proportionately low level of referrals to secondary care, high prevalence makes headache the most frequently listed reason for referral to neurologists and thus, uses capacity that is severely constrained. The potential decrease of referral rates to Neurology specialists as a result of direct access to imaging holds the potential release resources. On the other hand, direct access may be associated with a transfer of workload to Radiology, as not all patients directly referred to brain MRI would routinely undergo an MRI scan as part of the management of their chronic headache. This study aims to evaluate the healthcare utilisation and associated costs of GPs using direct access to brain MRI for the management of chronic headache patients compared with referral to a Neurologist.

\section{PATIENTS AND METHODS}

\section{Study design and patient selection}

The study was an independent single-centre site, prospective, observational study. The study compared two existing clinical pathways in the management of patients with a chronic headache from either GP referral to the Neurology Department or to direct access to brain MRI. No change to clinical practice was involved. Participants were allocated to two groups, the Neurology group or the MRI group. The allocation was decided a priori, that is, the referrer (in this case the GP) decided which referral route would suit each participant. Subsequent care was consistent with a standard of care for each clinical pathway and depicted in figure 1 .

Patients eligible for the study included adults aged 16 years or over with chronic migraine defined as occurring $\geq 15$ days per month for more than 3 months (as per International Classification of Headache Disorders 3 (ICHD-3) criteria) and referred from GP practices to a local Hospital in Central London, either for a Neurology outpatient appointment or an MRI examination. Participants were excluded if there were secondary causes for the headache, if they were prisoners, lacked the capacity to give consent or participate in the study, not fluent in English or already taking part in a clinical trial of an investigational medicinal product. Following eligibility assessment, participants completed a written informed consent.

\section{Patient and public involvement statement}

This study has involved patient and public involvement during the design and dissemination phase. Prior to the start of the research, a group of patients suffering from chronic headache were consulted to coproduce the study mobile app that was used to monitor symptoms and triggers of chronic headache. The patients who were involved in the study design, as well as all study participants, received a lay summary of the research findings as per their preferences, either via post or email. Research data are available on reasonable request.

\section{Study outcomes}

The primary outcome was to estimate the 6-month healthcare costs associated with two existing clinical pathways in the management of chronic headaches. Secondary outcomes comprised: the extension of the cost analysis up to 12 months, the evaluation of access to care, patient satisfaction, headache burden and time off work associated with both clinical pathways.

\section{Service use costs}

Total costs were calculated based on the multiplication of any headache-related healthcare events by the unit cost of each event. Resource use data included contacts with any NHS healthcare provider associated with the management of chronic headache. These included, among others, visits to GPs or headache clinical nurse specialist, inpatient care, neurologist or other headacherelated outpatient visits (eg, psychiatry), physiotherapist, 


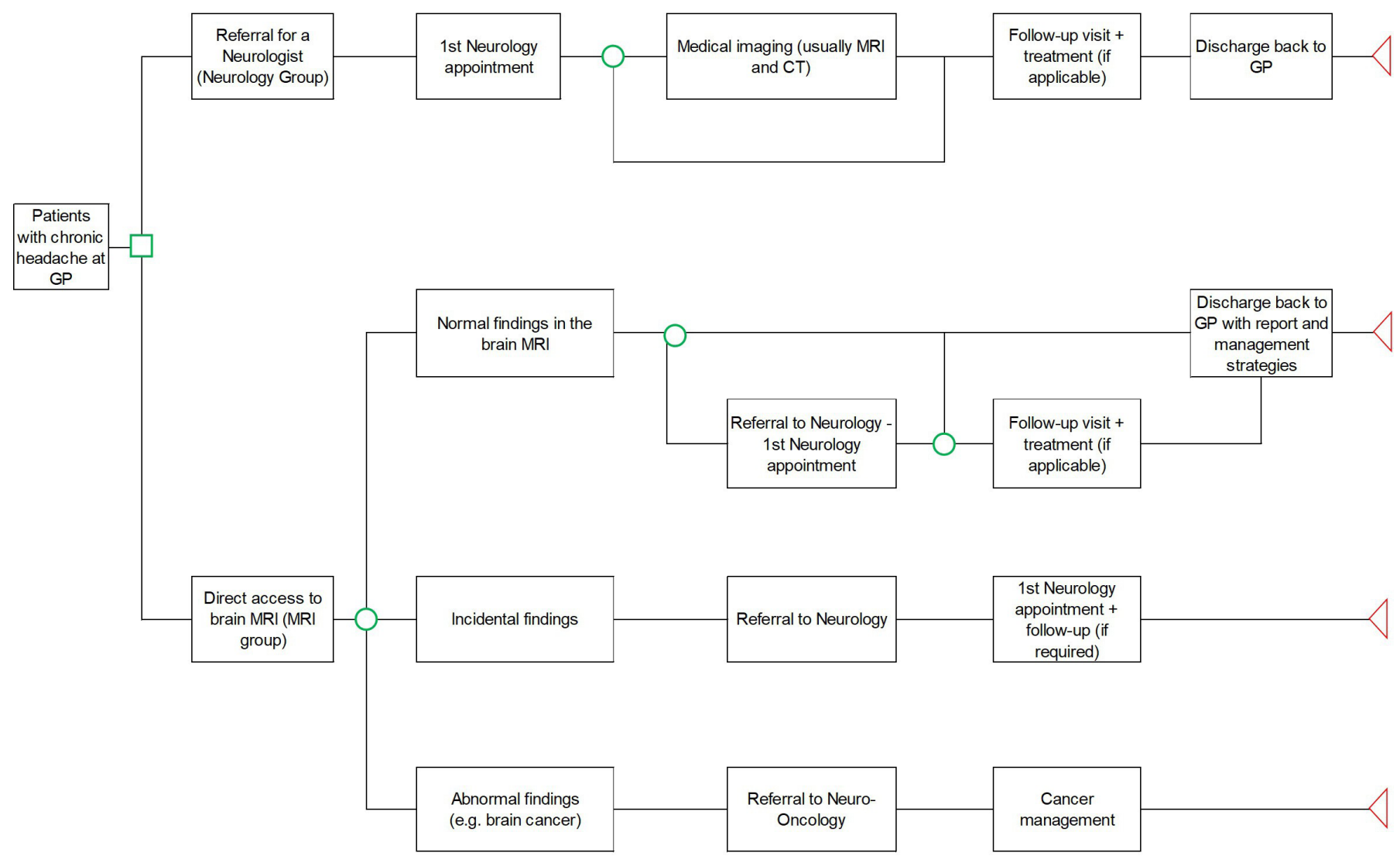

Figure 1 High-level illustration of two existing clinical pathways associated with the referral from GP due to chronic headache. GP, general practitioner.

visits to the ED, advanced imaging such as CT and MRI. Resource use data were retrieved from multiple hospital and primary care databases as well as self-reported data from participants using a participant headache diary (either paper-based or a headache mobile app). For the purposes of the primary outcome, the valuation of unit costs was, whenever possible, based on NHS Reference Costs 2016-17. ${ }^{22}$ Medication costs were derived from Prescription Cost Analysis ${ }^{23}$ and estimated from clinical data, specifically secondary care clinic letters and information provided by primary care. Participants were also asked to record time off work due to headache using the weekly participant diary.

\section{Quality of life and headache burden}

Headache burden was assessed using validated headache questionnaires and headache diaries. The HIT-6 questionnaire measured the headache burden based on six questions, leading to a score range from 36 to 78 . The Migraine Disability Assessment Scale (MIDAS) questionnaire assessed the disability associated with the headache (ranges from 0 to 90). Furthermore, along with the headache diaries completed by participants, the MIDAS questionnaire allowed the estimate of the number of headache days per quarter (maximum of 90 days) and self-reported headache pain scores (ranging from 0 , no pain at all, to 10 , the worst pain ever).

\section{Access to care}

The time elapsed between the GP referral and the first appointment in each group, either the Neurology appointment or the MRI scan, was measured as a proxy for access to care. Furthermore, in the MRI group, the availability of the MRI report to the GP, rather than the actual timing of the diagnostic scan, was considered.

\section{Change in care management}

Change in care management in both groups was evaluated. It was considered that a change in care management occurred when patients underwent new treatment options (medication or otherwise, eg, new headache medication, Botox treatment).

\section{Patient satisfaction}

Patient satisfaction was evaluated at month 3 postrecruitment using a non-validated questionnaire. This questionnaire evaluated three dimensions of analysis: (a) referral process (time elapsed between referral from primary care to initial secondary care appointment); (b) initial appointment and (c) overall experience 3 months after recruitment.

\section{Statistical analyses}

This study was observational but all analyses were based on the principle of 'intention-to-treat' so that participants recruited were included in the analysis 
as per the group they were recruited to, regardless of whether they actually received the intended treatment, any protocol deviations or potential losses to follow-up. ${ }^{24}$ Given the study's time horizon of 12 months, no discounting of costs or effects were considered. Descriptive statistics on several sociodemographics baseline characteristics were included. Differences in baseline characteristics between treatment groups were compared using statistical tests: $\chi^{2}$ for categorical data, t-test for Normal data and MannWhitney for non-Normal quantitative data. Given the skewness associated with the cost distribution, all cost differences between groups were assessed using generalised linear models (GLM) with an identity-link and gamma distribution. An identity link function instead of a $\log$ link was considered in order to avoid potential analytical biases. ${ }^{25}{ }^{26}$ An unadjusted GLM cost analysis with the study group (MRI group vs Neurology group) as only univariate analysis was performed as the first step. Given the study's observational design, the cost difference can be impacted due to the lack of randomisation. ${ }^{27}$ Baseline variables that differenced between the two groups $(\mathrm{p}<0.1)$ were included in the multivariable (adjusted) GLM analyses. For all GLM analyses, group difference estimates and associated confidence intervals were reported, together with $\mathrm{p}$ values. Analyses were conducted using Stata V.15.

\section{Sample size}

The sample size estimate was calculated based on the primary endpoint, total 6-month healthcare costs. A total of 150 participants were recruited in the Neurology group and 99 participants in the MRI group to achieve a detection a cost difference of $£ 300$ assuming SD of $£ 750$ and $£ 500$, respectively, with $85 \%$ power at the $5 \%$ twosided significance level. A $20 \%$ increase in sample size due to unknown cost distribution and attrition rate was considered.

\section{RESULTS}

A total of 249 participants were recruited, 150 in the Neurology group and 99 in the MRI group. In total, $100 \%(n=128)$ and $97 \%(n=96)$ of participants recruited received the treatment allocation in the Neurology and the MRI group, respectively (figure 2). With regards to the follow-up duration, $15 \%(\mathrm{n}=22)$ and $4.0 \% \quad(n=4)$ participants withdrew from the Neurology and MRI group, respectively, and were considered lost to follow-up. Remaining participants $(\mathrm{n}=223)$ were included in the analysis, equivalent to $128(85 \%)$ and $95(96 \%)$ participants in the Neurology and MRI group, respectively.

Baseline sociodemographic, clinical variables and resource use in the 12 months prerecruitment are detailed in table 1. A higher proportion of women were recruited to the Neurology group compared with the MRI group ( $81 \%$ vs $68 \%, \mathrm{p}=0.039)$. Both groups were

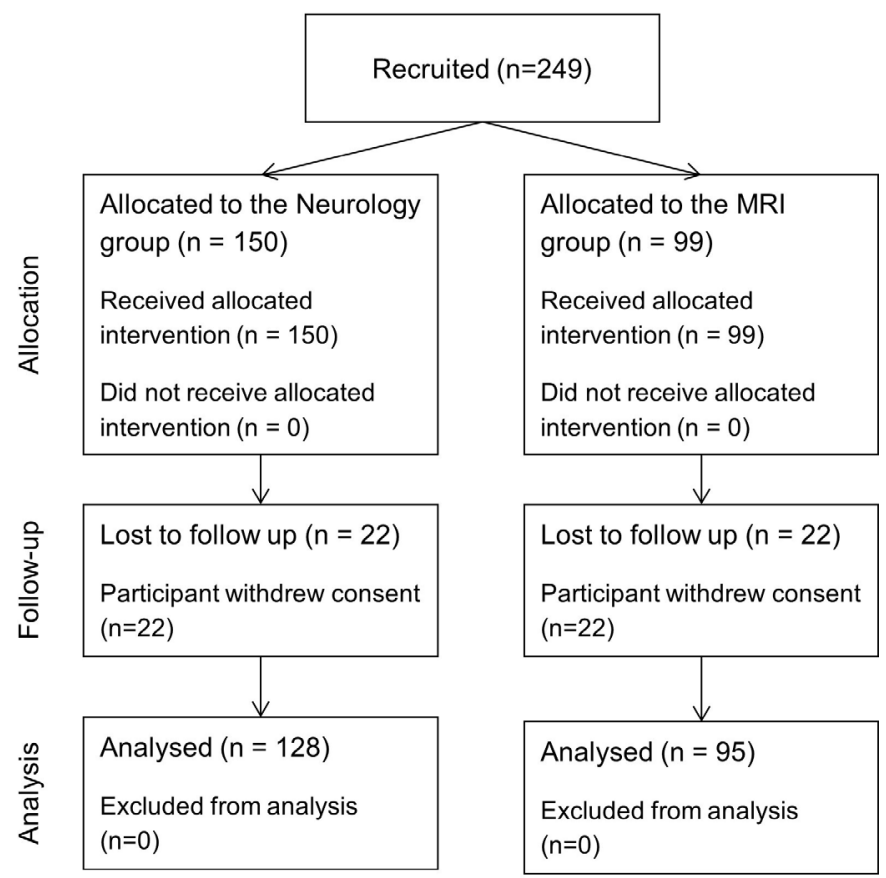

Figure 2 Participant flow chart for the headache study.

similar in mean age, ethnic mix, with more than half being White. No significant differences between the groups were found in terms of highest qualification and employment, with high-school and full-time employment being the most common. In terms of clinical variables, no significant difference between groups were found in terms of number of active health problems or number of headache triggers. Participants in the Neurology group reported lower utility and health scores using the generic 5-level EQ-5D (EQ-5D-5L) questionnaire, with a statistically significant difference in health scores $(p=0.005)$. Lower health scores imply lower self-rated quality of life, while for the headache-specific questionnaires (HIT-6 and MIDAS), a higher score implies a higher headache burden. Compared with the MRI group, participants in the Neurology group reported significantly higher headache burden (HIT-6 mean score: 65 vs $63, \mathrm{p}=0.006$ ), number of headache days in the past 3 months (MIDAS questionnaire: 52 vs $43, \mathrm{p}=0.038$ ). Participants in the Neurology group also had significantly more primary care appointments (3.7 vs 2.4, $\mathrm{p}<0.001)$ and all NHS appointments ( 4.3 vs $2.5, \mathrm{p}<0.001)$.

\section{Service use}

Table 2 summarises the NHS resource use of primary care and hospital-based services over a period of 12 months following recruitment. With regards to primary care utilisation, participants in the Neurology group had a significantly higher number of GP face-to-face appointments (mean number per participant: 1.82 vs $1.19, \mathrm{p}=0.006$ ). Regarding secondary care, participants in the Neurology group had a higher mean number of outpatient appointments $(2.52$ vs $0.26, \mathrm{p}<0.001)$ and other treatments such as Botox and nerve root injection (mean 0.30 vs $0.05, \mathrm{p}<0.001$ ). In contrast, 
Table 1 Baseline characteristics of the population analysed

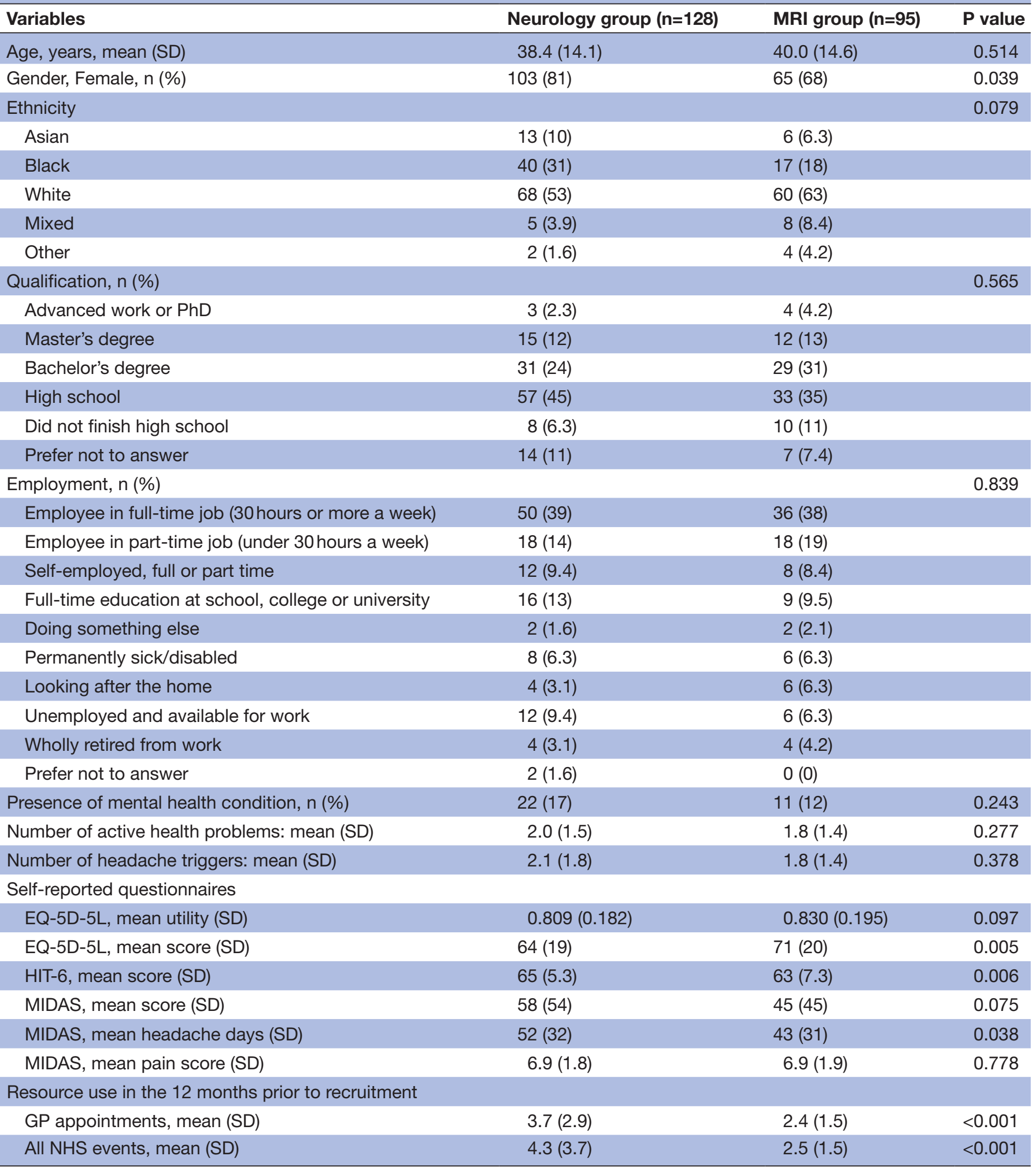

EQ-5D-5L, 5-level EQ-5D; GP, general practitioner; HIT-6, Headache Impact Test; MIDAS, Migraine Disability Assessment Scale; NHS, National Health Service.

they had a lower mean number of brain MRIs $(0.59 \mathrm{vs}$ $1.05, \mathrm{p}<0.001)$. There were no statistically significant differences in the utilisation levels of any of the other healthcare events.
The NHS resource use pre and postrecruitment for both groups was also compared. Table 3 summarises the difference between headache-related events 12 months postrecruitment compared with the 12 months prerecruitment. 
Table 2 Breakdown of number of NHS appointments per type of activity organised per group and respective number of participants responsible for these appointments (12 months postrecruitment)

\begin{tabular}{|c|c|c|c|c|c|c|c|}
\hline \multirow[b]{2}{*}{ Type of NHS appointment } & \multicolumn{3}{|c|}{ Neurology group $(n=128)$} & \multicolumn{3}{|c|}{ MRI group (n=95) } & \multirow[b]{2}{*}{$P$ value } \\
\hline & $\begin{array}{l}\text { Total of } \\
\text { episodes }\end{array}$ & Mean (SD) & N (\%) & $\begin{array}{l}\text { Total of } \\
\text { episodes }\end{array}$ & Mean (SD) & N (\%) & \\
\hline \multicolumn{8}{|l|}{ Primary care services } \\
\hline GP face-to-face appointment & 233 & $1.82(2.11)$ & $91(71)$ & 113 & $1.19(1.64)$ & $57(60)$ & 0.006 \\
\hline GP phone appointment & 37 & $0.29(0.75)$ & $24(19)$ & 25 & $0.26(0.49)$ & $23(24)$ & 0.420 \\
\hline \multicolumn{8}{|l|}{ Hospital-based services } \\
\hline Hospital outpatient appointment & 322 & $2.52(1.19)$ & $128(100)$ & 25 & $0.26(0.55)$ & $20(21)$ & $<0.001$ \\
\hline Inpatient episode & 4 & $0.03(0.35)$ & $1(0.8)$ & 1 & $0.01(0.10)$ & $1(1.1)$ & 0.837 \\
\hline Emergency department episode & 8 & $0.06(0.24)$ & $8(6.3)$ & 5 & $0.05(0.22)$ & $5(5.3)$ & 0.756 \\
\hline Head CT & 1 & $0.01(0.09)$ & $1(0.8)$ & 1 & $0.01(0.10)$ & $1(1.1)$ & 0.832 \\
\hline Brain MRI & 75 & $0.59(0.49)$ & $75(59)$ & 100 & $1.05(0.30)$ & 95 (100) & $<0.001$ \\
\hline $\begin{array}{l}\text { Others (eg, botox and nerve injection } \\
\text { treatments) }\end{array}$ & 39 & $0.30(0.79)$ & $25(20)$ & 5 & $0.05(0.30)$ & $3(3.2)$ & $<0.001$ \\
\hline
\end{tabular}

GP, general practitioner; NHS, National Health Service.

A reduction of 197 and 87 GP visits (mean 1.54/0.92 per participant or a percentage reduction of $85 \% / 77 \%$ ) was noted in the Neurology and MRI group, respectively. Similarly, there was a reduction in ED utilisation with a decrease of 23 and 6 episodes (mean 0.18 and 0.07 per participant or a percentage reduction of $288 \%$ and $120 \%$ ) in the Neurology and MRI group, respectively.

\section{Cost analyses}

The mean (SD) cost management per participant at 6 months postrecruitment was lower in the MRI group compared with the Neurology group ( 245 ( $£ 172)$ vs $£ 578$ (£420)), leading to a mean cost difference between groups of $-£ 333$ per participant (95\% CI: $-£ 413$ to $-£ 253$, $\mathrm{p}<0.001)$. The MRI group had a higher proportion of participants with lower costs ( $£ 0-£ 250$ range) than the Neurology group ( $73 \%$ vs $4.7 \%$ ). The initial cost analysis was extended to 12 months postrecruitment and the mean cost difference between groups increased, with the MRI group generating cost savings of $£ 518$ per participant $(95 \%$ CI: $-£ 637$ to $-£ 401, \mathrm{p}<0.001)$.

Given the non-randomised study design, an additional GLM analysis was performed to adjust for baseline characteristics. The unadjusted 6-month cost difference between groups (-£333; 95\% CI: $-£ 413$ to $-£ 253)$ hardly changed after adjustment (-£308; 95\% CI: $-£ 408$ to $-£ 209)$. In all analyses, at both 6 and 12 months postrecruitment, direct access to MRI for the management of chronic headache was associated with statistically significant mean cost savings for the NHS.

\section{Abnormal findings in the MRI group}

Out of the 95 participants recruited in the MRI group, three MRIs were not performed during the initial appointment

Table 3 Difference in the number, mean and percentage reduction of NHS appointments per type of activity organised per group 12 months postrecruitment compared with the 12 months prerecruitment (note: a negative/positive percentage denotes a decrease/increase in activity following recruitment)

\begin{tabular}{|c|c|c|c|c|c|c|}
\hline \multirow[b]{2}{*}{ Type of NHS appointment } & \multicolumn{3}{|c|}{ Neurology group $(n=128)$} & \multicolumn{3}{|l|}{ MRI group $(n=95)$} \\
\hline & Total of episodes & Mean & $\%$ & Total of episodes & Mean & $\%$ \\
\hline \multicolumn{7}{|l|}{ Primary care services } \\
\hline GP phone appointment & -11 & -0.09 & -30 & 5 & 0.05 & 20 \\
\hline \multicolumn{7}{|l|}{ Hospital-based services } \\
\hline Inpatient episode & 3 & 0.02 & 75 & 1 & 0.01 & 100 \\
\hline Emergency department episode & -23 & -0.18 & -288 & -6 & -0.07 & -120 \\
\hline Head CT & -9 & -0.07 & -12 & 1 & 0.01 & 1.0 \\
\hline Brain MRI & 55 & 0.43 & 141 & 100 & 1.05 & NA \\
\hline
\end{tabular}

GP, general practitioner; NHS, National Health Service. 
Table 4 Description of incidental findings, its clinical relevance and subsequent pathway

\begin{tabular}{lll}
\hline Abnormal findings & Significant (yes/no)? & Changes in diagnostic or treatment pathway \\
\hline Mature striatocapsular lacune & No & \\
$\begin{array}{l}\text { Sinusitis with complete opacification } \\
\text { Pituitary abnormality (T1) }\end{array}$ & No & Ear, nose, and throat (ENT) specialist review only \\
Low lying cerebellar tonsils & No & \\
Previous petrous surgery noted & No & Referred to neurovascular-no coiling (no intervention) \\
$\begin{array}{l}\text { Two aneurysms AcomA (anterior } \\
\text { communicating artery) and right ICA }\end{array}$ & Yes & Follow-up MRI only \\
\hline No definitive lesion & No &
\end{tabular}

ICA, internal carotid artery.

due to claustrophobia events. From a total number of 92 MRIs, $85(92 \%)$ were normal and $7(7.6 \%)$ presented abnormal findings (table 4), with one diagnosis being particularly significant (two small intracranial aneurysms). This participant was referred to neurovascular team for assessment, at which point no intervention was performed during the follow-up period (participant included in an active surveillance group). Two other participants had a change in their clinical management for less significant findings, leading to either a follow-up appointment or scan. No brain malignancies were diagnosed.

\section{Access to care}

The mean time (SD) elapsed from GP referral to the initial Neurology and MRI scan was, respectively, 110 (35) days and 39 days (17), a statistically significant difference $(p<0.001)$. Second, if the MRI report, than the actual scan, is considered as a proxy for access to care, the mean time elapsed was 70 days $(35)(\mathrm{p}<0.001)$.

\section{Change in management}

Participants in the Neurology group were more likely to have a change in therapeutic management compared with participants in the MRI group (97\% vs 64\%, p<0.001). Similarly, among participants that had not started on preventative medication prerecruitment, a higher proportion of participants in the Neurology group were started on preventative medication as part of their clinical management $(93 \%$ vs $53 \%, \mathrm{p}<0.001)$.

\section{Quality of life and headache burden}

At baseline, mean utility was lower in the Neurology group but not significant (mean utility of 0.809 vs 0.830 , $\mathrm{p}=0.097$ ), while their self-perceived mean health score was significantly lower (EQ-5D-5L: visual analogue scale score of 64.0 vs $70.8, \mathrm{p}=0.005$ ) (table 5 ). There was no statistically significant differences between the groups at 6 months for the utility and self-reported healthcare scores.

At baseline, participants in the Neurology group presented a higher headache burden compared with the MRI group (mean score of 65.0 vs 62.6, $\mathrm{p}=0.006$ ) (table 5). This trend seemed to be maintained over the follow-up period but was difficult to interpret due to high attrition rates: mean HIT-6 score at 6 months (60.0 vs 53.1, $\mathrm{p}=0.968$ ). At baseline, participants in the Neurology group had higher headache severity compared with the MRI group, but this was not significant (mean MIDAS score of 57.8 vs 44.8, $\mathrm{p}=0.075$ ) (table 5). In both groups, the headache severity decreased over the follow-up period. At baseline, participants in the Neurology group reported a significantly higher mean number of headache days compared with the MRI group (51.6 vs 42.8 , $\mathrm{p}=0.038)$. This trend was observed at month 6 but was not significant (41.2 vs $26.5, \mathrm{p}=0.152$ ). A third variable assessed self-reported headache pain scores. At baseline, participants at baseline showed almost identical mean headache pain scores (6.9 vs $6.9, \mathrm{p}=0.827)$. During the follow-up period, no statistical differences were found at month 6 ( 4.5 vs $4.2, \mathrm{p}=0.663)$.

\section{Time off work}

Participants in the Neurology group $(n=83)$ had a higher mean number of days off work due to headache compared with participants in the MRI group $(n=35)$ but these were not statistically significant at 6 (13.9 vs 9.7, $\mathrm{p}=0.563)$ or 12 months (27.9 vs 19.1, p=0.808) postrecruitment.

\section{Patient satisfaction}

Patient satisfaction was evaluated at month 3 postrecruitment using a non-validated questionnaire (online supplemental file 1). Participants in both groups reported no difference in terms of receiving an appointment in a suitable timeframe $(p=0.193)$. Participants in the MRI group reported higher satisfaction levels $(p=0.005)$ compared with the Neurology group associated with the information received prior to the actual appointment. No statistically significant difference $(p=0.366)$ between the groups was found regarding the satisfaction levels of both appointments (either MRI scan or Neurologist appointment) but a higher proportion of participants in the Neurology group reported a better experience compared with their expectation $(\mathrm{p}=0.002)$. At month 3 postrecruitment in all variables, except frequency of appointments $(\mathrm{p}=0.166)$, participants in the Neurology group reported higher levels of satisfaction with: amount of time spent with clinical staff ( $p=0.001)$; 
Table 5 Descriptive statistics for one generic questionnaire (EQ-5D-5L) and two headache-specific questionnaires (HIT-6 and MIDAS) at baseline and 6 months postrecruitment

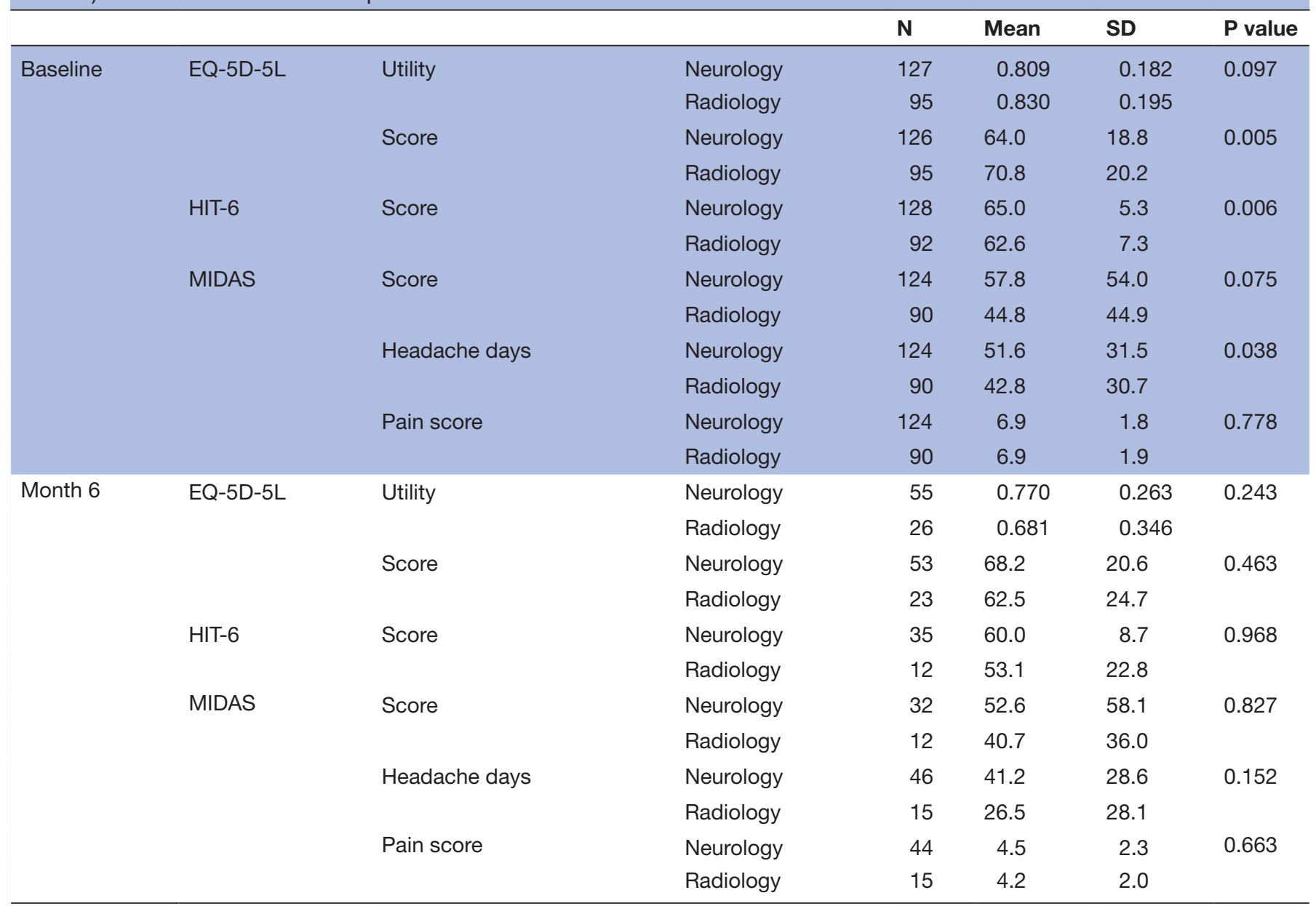

EQ-5D-5L, 5-level EQ-5D; HIT-6, Headache Impact Test; MIDAS, Migraine Disability Assessment Scale.

consistency of care $(\mathrm{p}=0.028)$; how informed you felt about your condition $(\mathrm{p}=0.010)$ /your treatment $(\mathrm{p}=0.004)$ and the overall experience $(\mathrm{p}<0.001)$.

\section{DISCUSSION}

Previous UK studies assessed the use of direct access to advanced imaging in the management of chronic headache patients. ${ }^{10}{ }^{21}$ Howard $e t a l^{21}$ performed a randomised controlled trial, which showed that the use of imaging in patients led to a reduction of referral rates to Neurology services in secondary care from $23 \%$ to $1.3 \%(1 / 76)$ in the treatment group (ie, patients being scanned). ${ }^{21}$ Thomas $e t a l^{10}$ estimated that direct access to brain imaging, in this case CT, reduced referral rates to Neurology in $86 \%$ of the cases during the follow-up period (average of 1.3 years per patient). ${ }^{10}$

The study's underlying hypothesis was that the early use of an advanced and accurate diagnostic tool (in this case MRI) would reassure both patients and GPs that no serious underlying cause (particularly brain tumour) is present. This would, in turn, reduce the headache burden and NHS resource use associated with the patient's subsequent management. Given the high prevalence of headache and the increased referral of patients with chronic headaches and other neurological conditions from primary care to hospital-based care, ${ }^{7}$ it is relevant to assess the implications of using different management strategies. To our knowledge, no previous prospective study in the UK has assessed the economic implications of these two coexisting management strategies based on GP referral decision.

The primary outcome was total costs at 6 months postrecruitment. The study showed that the use of advanced imaging produced cost-savings to the NHS compared with referral to Neurology, with mean cost-savings per participant of $£ 333$ and $£ 518$ at month 6 and 12 postrecruitment, respectively $(\mathrm{p}<0.001)$. These cost differences were multifactorial but primarily driven by: (1) the lower unit cost of a brain MRI scan (£146) compared with the initial Neurology appointment (£240); (2) the lower number of outpatient appointments in the MRI group (25 vs 322); (3) the fact that $75 / 128(59 \%)$ of participants in the Neurology group ended up having a brain MRI scan in the 12-month period of follow-up and (4) the increased 
likelihood of patients in the Neurology arm receiving management with Botox injections or nerve block procedures (39 vs 5 events). Direct access to brain MRI seemed to reassure most participants as only $17(18 \%)$ participants in the MRI group ended up being referred to a neurologist. Furthermore, over $66 \%$ of participants in the MRI group had no further hospital-based care, compared with $5 \%$ in the Neurology group. Similarly, at primary care level, participants in the MRI group presented lower utilisation rates per participant when compared with the Neurology group (mean GP appointments of 1.82 vs 1.19 , $\mathrm{p}=0.006$ ). In order to mitigate the potential confounding by indication introduced by GPs referring patients to both clinical pathways, adjusted GLMs were conducted. All cost differences between groups remained statistically significant $(p<0.001)$ when adjusted for differences in baseline characteristics or follow-up attrition rates.

At baseline, participants in the Neurology group presented lower self-reported quality of life and higher headache burden. HIT-6 scores did improve over time but we were unable to assess whether there was a statistically significant difference in both groups due to the high attrition rates, particularly in the MRI group. As mentioned, out of the 95 participants recruited to the MRI group, $17(18 \%)$ were subsequently referred to a Neurologist. Interestingly, at baseline, these 17 patients reported higher headache burden compared with all 95 patients recruited to the MRI group (MIDAS score: 51.2 vs 44.8; MIDAS headache days: 55.8 vs 42.8 ). This finding seems to suggest that these data might be useful to risk stratify patients and support GPs in their referral criteria. However, further research into this area is required.

Participants in the Neurology group presented a higher probability of being started on a preventative medication and had a change in therapeutic management following referral from primary care. A small proportion $(7.6 \%)$ of participants in the MRI group had abnormal findings in the initial brain MRI. Only one participant had clinically significant lesions (two small aneurysms) which were also incidental and no brain tumour was diagnosed.

Time off work was also evaluated as a proxy of headache burden. Participants in the Neurology group presented a trend ( $p>0.05)$ of a higher number of days off work due to headache compared with participants in the MRI group. This difference was not, however, statistically significant.

Lastly, patient satisfaction in both groups was compared based on three dimensions of analysis: during the referral period, the initial appointment and overall satisfaction. Twenty-five per cent of participants in the Neurology group (vs 18\% in the MRI group) reported dissatisfaction with the waiting time. This finding is not unexpected as the mean waiting time associated with the Neurology appointment was almost three times the one associated with the MRI scan (110 vs 39 days). Contrary to the referral period, participants in the Neurology group reported trends of higher satisfaction levels associated with the first appointment (neurology outpatient visit vs MRI scan) and a better experience compared with their expectations (60\% vs $29 \%$ in the Neurology and MRI group, respectively). Participants in the Neurology group reported improved satisfaction levels at 3 months across different variables (time spent with clinician, consistency of care, information about the condition and its treatment). Almost three quarters of Neurology participants reported being satisfied or very satisfied with their headache management compared with only $21 \%$ in the MRI group $(\mathrm{p}<0.001)$. Both participants and GP referrers in the MRI group reported dissatisfaction associated with the waiting time elapsed between the MRI scan and the availability of results (mean time of 31 days). This might have contributed to increased anxiety in some participants.

\section{Strengths of this study}

The estimate of NHS resource use data was primarily based on comprehensive and complete data retrieved from hospitalbased databases that captured both the acute and elective elements of the pathway associated with the management of patients with chronic headache. These data were supplemented by both primary care utilisation data, collected from each participant's GP and self-reported participant data. The aim was to guarantee that any chronic headache-related NHS event was costed regardless of the healthcare provider and its location. The prospective collection of healthcare utilisation and the evaluation of the impact of the interventions across different dimensions of analysis (efficiency, quality of care, access to care and patient satisfaction) were other key factors that contributed to the overall strength of the study.

\section{Limitations of this study}

There were some limitations to this study. First, this was a single-centre study with participants recruited from one central hospital in London. A multicentre study would be necessary to explore the generalisability of the results. Second, as with any observational study, no randomisation between groups was performed and there were significant differences in baseline of headache burden and health-scores and utilisation of resources. In order to mitigate potential confounding factors, adjusted multivariate analyses showed that the primary outcome was hardly affected and remained statistically significant. Nevertheless, residual confounding factors remain a limitation of the study. Third, specific inclusion and exclusion criteria were considered and as such, the study sample might not be representative of all patients with chronic headache. Fourth, for the purpose of secondary outcomes, most data were self-reported and hence prone to recall bias. Lastly, there were high follow-up attrition rates, particularly for participants in the MRI group, which affected some of the study's secondary outcomes.

\section{CONCLUSIONS}

This study found that the referral from primary care to direct access to brain MRI compared with referral for a neurologist for patients with chronic headache was associated with lower NHS overall costs at 6 and 12 months postrecruitment. Despite waiting longer from referral 
to appointment, participants in the Neurology group reported higher satisfaction levels associated with the care received compared with the MRI group and were more likely to benefit from changes to their therapeutic management.

\section{Implications for further research and clinical practice}

Baseline measures of headache burden, such as the HIT-6 or MIDAS, could potentially be used to determine which pathway may be suitable for patients but further research into the risk stratification of chronic headache patients is required. Future study designs should consider the confounding by indication introduced by recruiting participants referred to the two pathways based on GP decision. With regards to clinical practice, and based on the study findings, we plan to provide GPs with headache management advice along with the MRI report.

\section{Author affiliations}

${ }^{1}$ King's Health Economics, King's College London, London, UK

${ }^{2}$ Clinical Imaging and Medical Physics, Guy's and Saint Thomas' NHS Foundation Trust, London, UK

${ }^{3}$ Neuroradiology Group, Department of Radiology, Lambeth Wing, Guy's and St

Thomas' Hospitals NHS Foundation Trust, London, UK

${ }^{4}$ Neurology Department, Guy's and Saint Thomas' NHS Foundation Trust, London, UK

${ }^{5}$ Imaging Science Division, King's College London, London, UK

${ }^{6}$ Division of Primary Care and Public Health Sciences, King's College London,

London, UK

${ }^{7}$ School of Biomedical Engineering and Imaging Sciences, King's College London, London, UK

Acknowledgements The authors would like to thank all patients and staff members at Guy's and St Thomas' NHS Trust, particularly neurology and radiology consultants, for making this study possible.

Contributors TR, AM, YA, JT, RR, JLP, PM and SA contributed to conception and design of data. TR, YA, CM and JO contributed to acquisition of data. TR, JLP, PM and JS involved in analysis and interpretation of data. TR, AM, JLP, PM, VG, JS and SA involved in drafting the manuscript. TR, AM, YA, CM, JT, RR, JLP, PM, VG, JS and SA contributed to final approval of the completed manuscript.

Funding This work was supported by the Guy's and St Thomas' Charity.

Competing interests None declared.

Patient consent for publication Not required.

Ethics approval The Health Research Authority and Research Ethics Committee (West of Scotland-REC 4) approved the study research on 12 April 2016 (REC reference 16/WS/0028).

Provenance and peer review Not commissioned; externally peer reviewed.

Data availability statement Data are available upon reasonable request. Extra data can be accessed via the Dryad data repository at http://datadryad.org/ with the doi: $10.5061 /$ dryad.d7wm37pzn.

Open access This is an open access article distributed in accordance with the Creative Commons Attribution Non Commercial (CC BY-NC 4.0) license, which permits others to distribute, remix, adapt, build upon this work non-commercially, and license their derivative works on different terms, provided the original work is properly cited, appropriate credit is given, any changes made indicated, and the use is non-commercial. See: http://creativecommons.org/licenses/by-nc/4.0/.

ORCID iD

Tiago Rua http://orcid.org/0000-0002-2746-5663
REFERENCES

1 Jensen R, Stovner LJ. Epidemiology and comorbidity of headache. Lancet Neurol 2008;7:354-61.

2 Stovner L, Hagen K, Jensen R, et al. The global burden of headache: a documentation of headache prevalence and disability worldwide. Cephalalgia 2007;27:193-210.

3 Boardman HF, Thomas E, Croft PR, et al. Epidemiology of headache in an English district. Cephalalgia 2003;23:129-37.

4 Vos T, Flaxman AD, Naghavi M, et al. Years lived with disability (YLDs) for 1160 sequelae of 289 diseases and injuries 1990-2010: a systematic analysis for the global burden of disease study 2010 . The Lancet 2012;380:2163-96.

5 Leonardi M, Raggi A. Burden of migraine: international perspectives. Neurological Sciences 2013;34:117-8.

6 Rasmussen BK, Jensen R, Schroll M, et al. Epidemiology of headache in a general population--a prevalence study. $J$ Clin Epidemiol 1991:44:1147-57.

7 Latinovic R, Gulliford M, Ridsdale L. Headache and migraine in primary care: consultation, prescription, and referral rates in a large population. J Neurol Neurosurg Psychiatry 2006;77:385-7.

8 Department of Health. Direct access to diagnostic tests for cancer: best practice referral pathways for general practitioners, 2012.

9 Braschinsky M, Haldre S, Kals M, et al. Structured education can improve primary-care management of headache: the first empirical evidence, from a controlled interventional study. J Headache Pain 2016;17:24.

10 Thomas R, Cook A, Main G, et al. Primary care access to computed tomography for chronic headache. Br J Gen Pract 2010;60:426-30.

11 Patterson VH, Esmonde TF. Comparison of the handling of neurological outpatient referrals by general physicians and a neurologist. J Neurol Neurosurg Psychiatry 1993;56:830.

12 Ridsdale L, Doherty J, McCrone P, et al. A new GP with special interest headache service: observational study. Br J Gen Pract 2008;58:478-83.

13 Osumili B, McCrone P, Cousins S, et al. The economic cost of patients with migraine headache referred to specialist clinics. Headache 2018;58:287-94.

14 Bloudek LM, Stokes M, Buse DC, et al. Cost of healthcare for patients with migraine in five European countries: results from the International burden of migraine study (IBMS). J Headache Pain 2012;13:361-78.

15 Katsarava Z, Mania M, Lampl C, et al. Poor medical care for people with migraine in Europe - evidence from the Eurolight study. $J$ Headache Pain 2018;19:10.

16 McCrone P, Seed PT, Dowson AJ, et al. Service use and costs for people with headache: a UK primary care study. $J$ Headache Pain 2011;12:617-23.

17 Morgan M, Jenkins L, Ridsdale L. Patient pressure for referral for headache: a qualitative study of GPs' referral behaviour. $\mathrm{Br} J$ Gen Pract J R Coll Gen Pract 2007;57:29-35.

18 Underwood R, Kilner R, Ridsdale L. Primary care management of headaches and how direct-access MRI fits: a qualitative study of UK general practitioners' views. BMJ Open 2017;7:e018169.

19 NICE. Management of headaches, 2018. Available: https://pathways. nice.org.uk/pathways/headaches [Accessed 28 Jan 2019].

20 Callaghan BC, Kerber KA, Pace RJ, et al. Headache neuroimaging: routine testing when guidelines recommend against them. Cephalalgia 2015;35:1144-52

21 Howard L, Wessely S, Leese M, et al. Are investigations anxiolytic or anxiogenic? a randomised controlled trial of neuroimaging to provide reassurance in chronic daily headache. $J$ Neurol Neurosurg Psychiatry 2005;76:1558-64.

22 NHS Improvement. Reference costs | NHS improvement, 2017. Available: https://improvement.nhs.uk/resources/reference-costs/\# rc1718 [Accessed 31 Jul 2019].

23 NHS Business Services Authority. Prescription cost analysis (PCA) data, 2018. Available: https://www.nhsbsa.nhs.uk/prescription-data/ dispensing-data/prescription-cost-analysis-pca-data [Accessed 31 Jul 2019].

24 Gupta SK. Intention-To-Treat concept: a review. Perspect Clin Res 2011;2:109-12.

25 Polgreen LA, Brooks JM. Estimating incremental costs with skew: a cautionary note. Appl Health Econ Health Policy 2012;10:319-29.

26 Barber JA, Thompson SG. Analysis of cost data in randomized trials: an application of the non-parametric bootstrap. Stat Med 2000;19:3219-36.

27 Moran JL, Solomon PJ, Peisach AR, et al. New models for old questions: generalized linear models for cost prediction. $J$ Eval Clin Pract 2007;13:381-9. 